\title{
Pediatric Emergency Medicine Simulation Curriculum: Cardiac Tamponade
}

Julie A. Augenstein, MD, Jason Deen, MD, Anita Thomas, MD, MPH, Emily Moser, MD, Kimberly Stone, MD, Jennifer Reid, MD, Rebekah Burns, MD*

*Corresponding author: rebekah.burns@seattlechildrens.org

\section{Abstract}

Introduction: Cardiac tamponade is an uncommon presentation to the pediatric emergency department and requires early recognition and emergent intervention. Methods: We developed this patient simulation case to simulate a low-frequency, high-acuity scenario for pediatric emergency medicine fellows and resident physicians in emergency medicine, pediatrics, and family medicine. We ran the case in a pediatric emergency department using a high-fidelity pediatric mannequin and equipment found in the clinical environment, including a bedside ultrasound machine. The case involved a 10-year-old patient with Hodgkin lymphoma who presented with fever, neutropenia, and shock and was found to have a pericardial effusion with tamponade after evaluation. The providers were expected to identify signs and symptoms of shock, as well as cardiac tamponade, and demonstrate appropriate emergent evaluation and management. Required personnel included a simulation technician, instructors, and a nurse. Debriefing tools tailored specifically for this scenario were created to facilitate a formal debriefing and formative learner assessment at the end of the simulation. Results: This case has been implemented with 10 pediatric emergency medicine fellows during two 3-year cycles of fellow education. Session feedback reflected a high level of satisfaction with the case and an increased awareness of bedside ultrasound in the identification of cardiac tamponade. Discussion: This resource for teaching the critical components for diagnosing and managing unstable cardiac tamponade in the pediatric patient, including use of bedside ultrasound, was well received by pediatric emergency medicine fellows.

\section{Keywords}

Ultrasound, Shock, Tamponade, Fever and Neutropenia, Pediatric Simulation

\section{Educational Objectives}

By the end of this module, learners will be able to:

1. Perform an initial evaluation and stabilization of an immunocompromised patient with fever and shock.

2. Describe signs and symptoms concerning for cardiac tamponade.

3. Demonstrate management of an unstable patient with cardiac tamponade.

4. List the findings on a limited point-of-care four-view cardiac ultrasound that are concerning for tamponade (optional).

5. Demonstrate effective team communication with members of the health care team.

\section{Introduction}

Cardiac tamponade is an infrequent but life-threatening diagnosis in the pediatric emergency department. Given the need for emergent intervention to improve prognosis and attenuate mortality, it is imperative to identify this condition rapidly. This simulation scenario allows learners to develop an approach to the diagnosis of cardiac tamponade, practice interpretation of bedside cardiac ultrasound images, and stabilize an acutely ill patient. Required prerequisite general knowledge includes the ability to rapidly assess and stabilize a critically ill patient, an understanding of the signs and symptoms of cardiac tamponade, and technical skill in the use and interpretation of point-of-care ultrasound (POCUS).

Citation: Augenstein JA, Deen J, Thomas A, et al. Pediatric Emergency Medicine Simulation Curriculum: cardiac tamponade. MedEdPORTAL. 2018;14:10758.

https://doi.org/10.15766/mep_23748265.10758

Copyright: $\odot 2018$ Augenstein et al. This is an open-access publication distributed under the terms of the Creative Commons AttributionNonCommercial license.

\section{Appendices}

A. Tamponade Simulation Case.docx

B. Tamponade Simulation Environment Preparation docx

C. Tamponade CXR and ECG .pptx

D. Tamponade POC Echocardiogram Video folder

E. Tamponade TeamSTEPPS Glossary.docx

F. Tamponade Debriefing Materials.docx

G. Tamponade Simulation Evaluation Form.docx

H. Tamponade PowerPoint Presentation.pptx

All appendices are peer reviewed as integral parts of the Original Publication. 
Cardiac tamponade is a type of obstructive shock that is caused by cardiac compression due to fluid or gas in the pericardial space. Cardiac compression occurs once the pericardium has reached its stretching capacity, either due to intrapericardial volume or loss of pericardial elasticity. The causes of cardiac tamponade are varied and include effusion, trauma, and cardiac rupture. ${ }^{1}$ Pericardial effusions may be idiopathic or iatrogenic, and common etiologies include malignancy, uremia, infection, collagen vascular disease, hypothyroidism, and postacute myocardial infarction. ${ }^{2}$

The presentation of cardiac tamponade includes hypotension, tachypnea, pleuritic chest pain, and dyspnea. ${ }^{1}$ Physical exam may reveal a pericardial rub due to inflammatory effusions, quiet heart sounds, pulsus paradoxus (a decrease in systolic blood pressure and pulse wave amplitude during inspiration greater than $10 \mathrm{~mm} \mathrm{Hg}$ ), and jugular venous distension. ${ }^{3}$ Electrical alternans (changing QRS amplitudes) on electrocardiogram (ECG) is also suggestive of cardiac tamponade. ${ }^{3}$ A 2003 task force of the American College of Cardiology, American Heart Association, and American Society of Echocardiography recommended the use of echocardiography for patients with suspected pericardial disease and in the diagnosis of cardiac tamponade. ${ }^{4}$ Two-dimensional echocardiogram demonstrates a prominent fluid layer between the endocardium and pericardial sack, compressed cardiac chambers (typically the right atrium and right ventricle), high ejection fractions, and respiratory variation in atrioventricular valve inflow. 1,5,6

Simulation with structured debriefing is a specific teaching strategy that allows participants to develop clinical and communication tools in a safe environment. It facilitates experiential learning, as outlined by Kolb. ${ }^{7}$ Learners may engage in a concrete experience of clinical reasoning and patient care during this simulation, followed by reflective observation and abstract conceptualization during the debrief. Learners then participate in active experimentation by planning what they would do next time or employing skills during their next simulated or real-life experience. Pediatric emergency medicine (PEM) fellows have infrequent opportunities to participate in actual resuscitations. ${ }^{8}$ Therefore, preparation for infrequent but serious events can be, at least in part, met through simulation, with most PEM fellows now participating in simulation-based training during their fellowship. ${ }^{9}$

Although there has been a previous MedEdPORTAL publication of a simulation related to tamponade in a pediatric patient, ${ }^{10}$ the current simulation case targets additional learning objectives, including evaluating and managing shock in a febrile, neutropenic patient and interpreting a limited bedside cardiac POCUS. In addition, this simulation case includes chest radiograph, ECG, and ultrasound images, as well as a didactic slide deck and debriefing guide, all specifically tailored to the case. These components allow other educators to implement the case without seeking additional resources. This simulation-based case is particularly suited for physicians who encounter critically ill children in an emergent setting as it lets learners perform a primary and secondary survey in a hemodynamically unstable pediatric patient, develop and critically evaluate a differential diagnosis, and select appropriate management. The case was designed to teach learners to actively recall and utilize prior knowledge while in a realistic, high-acuity clinical setting. It can help identify knowledge gaps in the identification and management of patients with cardiac tamponade and provide further resources to advance learners' clinical skills. This case can be used independently or in conjunction with others from the Pediatric Emergency Medicine Simulation Curriculum. ${ }^{11-24}$

\section{Methods}

Development

This high-fidelity patient simulation case was developed by a PEM physician and a pediatric cardiologist to help learners recognize the critical components for diagnosing and managing unstable cardiac tamponade in a pediatric patient presenting with febrile neutropenia. The case was developed for PEM fellows but would also be appropriate for an audience of pediatric, family medicine, and emergency medicine (EM) residents; PEM and EM faculty; advanced practitioners; nurses; and intensive care unit trainees, nurses, and faculty. We chose to use a case of an immunocompromised patient to help emphasize early recognition of shock in a population frequently encountered in the pediatric emergency department 
setting and to reinforce the importance of rapid clinical management regardless of underlying etiology. As the differential diagnosis for immunocompromised patients with shock is wide, this case highlights the importance of using a systematic approach in the initial evaluation to determine the underlying etiology and appropriate management. Participants may be given additional learning materials before participation in this simulation depending on their backgrounds and educational needs. Prerequisite knowledge includes identification and management of obstructive shock and basic skills in POCUS (if ultrasound images are to be used). ${ }^{25,26}$

\section{Equipment/Environment}

The setting for this simulation case was the emergency department. Using a high-fidelity mannequin, we conducted the simulation in situ in the pediatric emergency department, but it could also be performed in a simulation lab or in the pediatric intensive care unit. At the start of the simulation, participants were informed that an immunocompromised patient with fever had just been taken to an exam room by the triage nurse. The bedside nurse attached the mannequin to monitors with vital signs as described in the simulation scenario (Appendix A). Changes in vital signs were demonstrated on the monitor using the standard software for SimJunior by Laerdal. See the simulation environment preparation document (Appendix B) for equipment and medications that were available. Diagnostic modalities including chest Xray (CXR) images, ECG, and bedside cardiac ultrasound were available to the learners if requested (Appendices C \& D). All images including video clips were created by the authors. An ultrasound machine was also available but was not essential for the simulation. If the learners' usual environment does not have bedside ultrasound, this piece may be omitted from the simulation case.

Adaptation if using low-fidelity simulator: Facilitators may verbally provide vital signs or use a simulator app for a phone or tablet. Facilitators may also describe the physical exam as the learners examine the mannequin.

\section{Personnel}

Required personnel needed to successfully implement this case included a simulation technician and facilitators. PEM physicians served as the facilitators at our institution. Four to six fellows participated in each simulation. However, the case can be modified to accommodate between three and eight participants. Ideally, the participants should function in their expected roles within the health care team (i.e., the physician roles are performed by physicians, and nurses are assigned nursing roles); however, due to personnel constraints at our institution, a physician facilitator played the role of the nurse for these sessions. If a second facilitator is available, he or she should play the role of the parent in order to provide history. The target number of participants should reflect the typical health care team at the participants' institution. Trainees or confederates can act in any unfilled roles if necessary, although the realism of the simulation may be compromised.

Implementation

This session was implemented during standard fellow education time. The simulation ran for approximately 20 minutes, with 40 minutes devoted to the debrief. The debrief occurred in a separate room from the simulation so that participants could focus on the discussion. The scenario began by telling the participants that a 10-year-old boy with Hodgkin lymphoma had arrived in the emergency department because of a low-grade fever at home. His history was also notable for 2 weeks of fatigue and shortness of breath. Participants were expected to begin an evaluation of neutropenic fever and identify that the patient was in shock. The patient's vital signs and exam were consistent for shock, prompting learners to consider possible causes and initiate management such as broad-spectrum antibiotics. His exam was notable for muffled heart sounds, and ECG and CXR demonstrated findings concerning for a pericardial effusion. Images demonstrating tamponade were provided to participants who requested a bedside cardiac ultrasound. The case ended when the learners identified the effusion and made plans to emergently consult cardiology. 
The facilitator (a PEM physician with training in simulation and medical education) and simulation technicians utilized the simulation case file to plan and execute the simulation scenario using a highfidelity mannequin. (See above for suggestions regarding use of low-fidelity options.) The simulation environment document was used beforehand to ensure that all required equipment was available. Ideally, this case should be adapted depending on what resources are actually available in the participants' usual work environment. Appendix C contains images, including an ECG and CXR, that were provided to participants during the simulation if they requested these studies. The time delay between their request and receiving the images should mirror that of practice in real life, when possible. Occasionally, due to time constraints, this lag time may need to be shortened. Appendix D contains video clips of a transthoracic cardiac ultrasound that were provided to participants for review and interpretation, if requested. The trainees had to demonstrate proper placement of the ultrasound probe on the patient prior to receiving the ultrasound images.

Appendix $E$ is a glossary of teamwork and communication terms that was reviewed by facilitators prior to leading the simulation. ${ }^{27}$ This helps create a shared language around leadership, teamwork, and communication and can also be used as an educational resource for participants prior to the simulation or by the facilitator to help guide discussion on teamwork and communication. Please see the Debriefing section below for specific instructions regarding the use of materials in Appendix $F$ during the debrief. Also included is an evaluation form (Appendix $G$ ) that was distributed to participants after the simulation and debrief in order gather feedback on the session and assess whether learning objectives were met, as perceived by the learners. The PowerPoint presentation (Appendix $\mathrm{H}$ ) is a short set of slides that provide a brief review of the etiologies, pathophysiology, evaluation, and management of cardiac tamponade. We used this presentation to lead a brief didactic session after the general debriefing session, but it could also be used as a review before the case, depending on the particular goals and objectives of the session.

Debriefing

Debriefing tools were tailored specifically for this scenario to facilitate a formal debriefing session and learner assessment at the end of the simulation. The Medical Management Evaluation/Debriefing Form and Teamwork and Communication Evaluation were used as a guide to lead the debriefing and provide formative feedback to learners after the session. These resources identify the critical components for diagnosing and managing unstable cardiac tamponade in a pediatric patient presenting with febrile neutropenia as well as crucial elements required for effective teamwork and communication.

The debriefing serves as one of the most valuable components of the session. As a general rule, we recommend allowing twice as much time for the debriefing as the simulation session, when possible. Ideally, participants and facilitators should debrief in a separate location away from the simulator to help focus discussion. We commenced the debrief by asking participants, "How did that feel?" This allowed the fellows to begin with a discussion of emotions that may have accompanied the simulation. Subsequently, participants naturally began a conversation about the medical management or diagnostic process. Using the debriefing guide, facilitators directed discussion about successes and challenges encountered during the simulation. Similarly, they ensured that communication skills, including leadership and team communication, were discussed.

Assessment

We created the Simulation Session Evaluation Form (Appendix G) to obtain feedback and evaluation on the simulation session in order to ensure that learners' needs were met and to provide feedback for improvement on future sessions. The form was revised for the second iteration of the session to quantify participants' self-assessment of their ability to meet the simulation's learning objectives.

During the debrief, participants received formative feedback and engaged in self-reflection regarding demonstration of knowledge, skills, and behaviors in the course of the simulation. Appendix F's Teamwork and Communication Evaluation was used to guide discussion. 


\section{Results}

This simulation case was used at our institution as part of the simulation and ultrasound curriculum for PEM fellows at various stages of their fellowship training (PGY 4-PGY 6). There are two to three fellows per year of the 3-year fellowship. The full simulation curriculum is 3 years in length so that no fellow repeats the same case twice. This simulation has been successfully implemented within our fellowship during two cycles of the curriculum, with a total of 10 PEM fellow participants thus far. We received extremely positive feedback from all participating trainees based on learner satisfaction data obtained from collected feedback forms (Table 1).

Table 1. Participant Feedback on Simulation as Part of the Pediatric Emergency Medicine Simulation Curriculum

Statement $M$ Score
$(n=10)^{\mathrm{a}}$

This simulation case provided is relevant to my work. $\quad 4.9$

The simulation case was realistic.

This simulation case was effective in teaching basic resuscitation skills.

This simulation case was effective in teaching cardiac tamponade management skills. $\quad 5.0$

The debrief promoted reflection and team discussion.

${ }^{\mathrm{a} O n}$ a 5 -point Likert scale $(1=$ strongly disagree, $3=$ neutral, 5 = strongly agree $)$.

With the second iteration of this curriculum, participants were asked to assess their confidence in their ability to perform key skills tasks directly related to the educational objectives (Table 2 ).

Table 2. Participant Assessment of Self-Confidence Related to Learning Objectives

\begin{tabular}{lr} 
Statement & $\begin{array}{c}\text { MScore } \\
(\boldsymbol{n}=6)^{a}\end{array}$ \\
\hline After participating in this session, how confident are you in your ability to: & 4.8 \\
Perform an initial evaluation and stabilization of an immunocompromised patient with fever and shock. & 4.8 \\
Describe signs and symptoms concerning for a cardiac tamponade. & 4.3 \\
Demonstrate management of an unstable patient with cardiac tamponade. & 4.3
\end{tabular}

aOn a 5-point Likert scale ( 1 = strongly disagree, $3=$ neutral, 5 = strongly agree).

Through feedback forms, participating trainees reported that their clinical practice changed due to the knowledge and skills obtained through the simulation; notably, the ability to identify and manage cardiac tamponade in an unstable pediatric patient improved as a direct outcome of participation. Trainees reported that they considered obtaining an ECG and performing a limited bedside cardiac POCUS when evaluating a patient with hypotension, tachycardia, and abnormal cardiac exam. Participants also reported that they were able to list the classic exam and diagnostic findings of cardiac tamponade after participation. In subsequent simulations in the curriculum, facilitators observed a positive trend in fellows incorporating bedside cardiac ultrasound into other scenarios. Of note, several participants suggested adding a procedural element to the scenario using a task trainer to actually perform a bedside pericardiocentesis for future iterations.

\section{Discussion}

This simulation-based educational resource was well received by PEM fellows and allowed learners to build and reinforce knowledge and skills in managing an unstable pediatric patient with cardiac tamponade by engaging them in the process of identification of the illness and expedient management. This high-fidelity patient simulation case was created to simulate a low-frequency but high-acuity scenario for PEM fellows and resident physicians in EM, pediatrics, and family medicine. Participation may help identify knowledge deficits in the identification and management of patients in obstructive shock secondary to cardiac tamponade. This case was created with the intention of being a comprehensive tool so that users would have access to all potential resources needed to implement the session in their own setting. The simulation scenario includes cues so that the instructor can provide relevant information and answer questions as appropriate during the session. The debriefing guide can help facilitators lead discussions to address any learning gaps or issues that may arise while allowing for flexibility depending on the specific learning objectives for a particular session. We have found that it is difficult to address 
more than three issues or topics during each debriefing due to time constraints but have included more than that number of options here so that resources are available depending upon what is most relevant for a given learner group.

A limitation noted while implementing this simulation is the challenge of incorporating bedside POCUS using the high-fidelity simulator mannequin. Using a model that has an ultrasound simulation feature or adding an adjunct to the scenario that could be used to obtain case-appropriate ultrasound images would increase realism and facilitate correct image acquisition and probe placement. An additional limitation identified during implementation is effectively illustrating a patient with worsening cardiac function so as to adequately reflect a clinically unstable patient and critical situation. To maintain the realism of the case, the facilitator has to reemphasize that the simulation patient is experiencing worsening shortness of breath following fluid administration or when placed in a supine position, as this cannot be adequately conveyed by the equipment capabilities. We also determined that telling participants the patient has distended jugular veins led them to the diagnosis quickly. In future iterations, we will therefore omit this detail unless specifically asked.

There are also limitations in the outcomes assessment for this simulation. First, our sample size was very small. We were limited by the number of fellows enrolled in our institution, as well as by scheduling factors that meant not every fellow was available on a given educational day. Second, we did not assess changes in learner knowledge or skill performance beyond what was discussed during the debriefing. Given that resuscitations generally and tamponade specifically are rare occurrences in PEM, directly assessing participant performance outside of the sessions is beyond the scope of our current educational capacity, as is assessing patient outcomes.

Since implementing this simulation, we have modified the participant feedback form so that we may better assess whether we are adequately addressing the learning objectives with our participants. In the next iteration, we plan on incorporating a task trainer so that participants may practice an emergent pericardiocentesis in the PED.

Julie A. Augenstein, MD: Pediatric Emergency Medicine Physician, Phoenix Children's Hospital

Jason Deen, MD: Assistant Professor, Department of Pediatrics, University of Washington School of Medicine

Anita Thomas, MD, MPH: Assistant Professor, Department of Pediatrics, University of Washington School of Medicine

Emily Moser, MD: Pediatrician, Kaiser Permanente, Colorado

Kimberly Stone, MD: Associate Professor, Department of Pediatrics, University of Washington School of Medicine

Jennifer Reid, MD: Associate Professor, Department of Pediatrics, University of Washington School of Medicine

Rebekah Burns, MD: Assistant Professor, Department of Pediatrics, University of Washington School of Medicine

\section{Disclosures}

None to report.

Funding/Support

None to report.

\section{Ethical Approval}

Reported as not applicable.

\section{References}

1. Spodick DH. Acute cardiac tamponade. N Engl J Med. 2003;349(7):684-690. https://doi.org/10.1056/NEJMra022643

2. Levy P-Y, Corey R, Berger P, et al. Etiologic diagnosis of 204 pericardial effusions. Medicine (Baltimore). 2003;82(6):385-391. https://doi.org/10.1097/01.md.0000101574.54295.73 
3. Spodick DH. Pericardial rub: prospective, multiple observer investigation of pericardial friction in 100 patients. Am J Cardiol. 1975;35(3):357-362. https://doi.org/10.1016/0002-9149(75)90027-2

4. Cheilin MD, Armstrong WF, Aurigemma GP, et al. ACC/AHA/ASE 2003 guideline update for the clinical application of echocardiography: summary article: a report of the American College of Cardiology/American Heart Association Task Force on Practice Guidelines (ACC/AHA/ASE Committee to Update the 1997 Guidelines for the Clinical Application of Echocardiography) Circulation. 2003;108(9):1146-1162. https://doi.org/10.1161/01.CIR.0000073597.57414.A9

5. D'Cruz I, Rehman AU, Hancock HL. Quantitative echocardiographic assessment in pericardial disease.Echocardiography 1997;14(2):207-213. https://doi.org/10.1111/j.1540-8175.1997.tb00712.x

6. Reydel B, Spodick DH. Frequency and significance of chamber collapses during cardiac tamponade. Am Heart J. 1990;119(5):1160-1163. https://doi.org/10.1016/S0002-8703(05)80248-0

7. Kolb DA. Experiential Learning: Experience as the Source of Learning and Development Upper Saddle River, NJ: Prentice Hall; 1983.

8. Mittiga MR, Geis GL, Kerrey BT, Rinderknecht AS. The spectrum and frequency of critical procedures performed in a pediatric emergency department: implications of a provider-level view. Ann Emerg Med. 2013;61(3):263-270. https://doi.org/10.1016/j.annemergmed.2012.06.021

9. Doughty CB, Kessler DO, Zuckerbraun NS, et al. Simulation in pediatric emergency medicine fellowships. Pediatrics. 2015;136(1):e152-e158. https://doi.org/10.1542/peds.2014-4158

10. Panesar R, Parker M, Messina CR, Gallagher C. Simulation interest group scenario: CJ with cardiac tamponade. MedEdPORTAL. 2012;8:9158. https://doi.org/10.15766/mep_2374-8265.9158

11. Reid J, Stone K. Pediatric Emergency Medicine Simulation Curriculum: hypovolemic shock. MedEdPORTAL. 2013;9:9452 https://doi.org/10.15766/mep_2374-8265.9452

12. Reid J, Stone K. Pediatric Emergency Medicine Simulation Curriculum: anaphylaxis. MedEdPORTAL. 2013;9:9638. https://doi.org/10.15766/mep_2374-8265.9638

13. Reid J, Stone K. Pediatric Emergency Medicine Simulation Curriculum: septic shock. MedEdPORTAL. 2013;9:9639. https://doi.org/10.15766/mep_2374-8265.9639

14. Reid J, Stone K. Pediatric Emergency Medicine Simulation Curriculum: status asthmaticus. MedEdPORTAL. 2014;10:9660 https://doi.org/10.15766/mep_2374-8265.9660

15. Stone K, Reid J. Pediatric Emergency Medicine Simulation Curriculum: supraventricular tachycardia. MedEdPORTAL. 2014;10:9716. https://doi.org/10.15766/mep_2374-8265.9716

16. Reid J, Stone K. Pediatric Emergency Medicine Simulation Curriculum: seizure scenario. MedEdPORTAL. 2014;10:9794. https://doi.org/10.15766/mep_2374-8265.9794

17. Reid J, Stone K. Pediatric Emergency Medicine Simulation Curriculum: ventricular fibrillation. MedEdPORTAL. 2014;10:9888. https://doi.org/10.15766/mep_2374-8265.9888

18. Uspal N, Stone K, Reid J, Coleman-Satterfield TT. Pediatric Emergency Medicine Simulation Curriculum: bronchiolitis MedEdPORTAL. 2015;11:10012. https://doi.org/10.15766/mep_2374-8265.10012

19. Reid J, Stone K, Otjen J. Pediatric Emergency Medicine Simulation Curriculum: blunt abdominal trauma. MedEdPORTAL. 2015;11:10013. https://doi.org/10.15766/mep_2374-8265.10013

20. Burns R, Stone K, Reid J, Malik F, Cheng A. Pediatric Emergency Medicine Simulation Curriculum: thyroid storm. MedEdPORTAL. 2015;11:10062. https://doi.org/10.15766/mep_2374-8265.10062

21. Reid J, Stone K. Pediatric Emergency Medicine Simulation Curriculum: traumatic brain injury. MedEdPORTAL. 2015;11:10067. https://doi.org/10.15766/mep_2374-8265.10067

22. Schuh A, Burns R, Reid J, Stone K. Pediatric emergency medicine simulation: hyperkalemia due to congenital adrenal hyperplasia. MedEdPORTAL. 2015;11:10250. https://doi.org/10.15766/mep_2374-8265.10250

23. Chua W, Burns R, Stone K, Reid J. Pediatric Emergency Medicine Simulation Curriculum: hyponatremic seizures. MedEdPORTAL. 2016;12:10498. https://doi.org/10.15766/mep_2374-8265.10498

24. Thomas A, Sanseau E, Uspal N, et al. Pediatric Emergency Medicine Simulation Curriculum: submersion injury with hypothermia and ventricular fibrillation. MedEdPORTAL. 2017;13:10643. https://doi.org/10.15766/mep_2374-8265.10643

25. Smith AT, Watnick C, Ferre RM. Cardiac tamponade diagnosed by point-of-care ultrasound. Pediatr Emer Care. 2017;33(2):132134.

26. Farah MM, Tay K-Y, Lavelle J. A general approach to ill and injured children. In: Shaw KN, Bachur RG, eds. Fleisher \& Ludwig's Textbook of Pediatric Emergency Medicine. 7th ed. Philadelphia, PA: Wolters Kluwer; 2016:1-8.

27. TeamSTEPPS. Agency for Healthcare Research and Quality website. http://teamstepps.ahrq.gov. Accessed February $23,2018$.

Received: April 3, 2018 | Accepted: September 1, 2018 | Published: September 28, 2018 